\title{
Monte Carlo model for pp, pA and AA collisions at high energy: parameters tuning and results
}

\author{
Vladimir Kovalenko* \\ Saint Petersburg State University \\ E-mail: nvkinferambler.ru
}

\begin{abstract}
The soft processes in pp, pA and AA collisions are considered in the Monte Carlo model with color string formation and fusion. The elementary nucleon collisions in the model are performed on the partonic level without usual assumptions of Glauber picture. The particle production is described in string model taking into account the interaction of strings in transverse plane in the approach of string fusion. Energy dependence of total inelastic cross section and mean charged multiplicity in pp collisions and recent $\mathrm{p}-\mathrm{Pb}$ data on multiplicity at $5.02 \mathrm{TeV}$ are used for the parameters turning. The comparison with experiment of the dependence of multiplicity per participant on the centrality in $\mathrm{PbPb}$ collisions at $2.76 \mathrm{TeV}$ shows that good agreement is obtained only when the string fusion effects are present with the radius of string $0.2-0.3 \mathrm{fm}$. The rapidity distribution of multiplicity in $\mathrm{PbPb}$ collisions is calculated and compared with experimental data.
\end{abstract}

The XXI International Workshop High Energy Physics and Quantum Field Theory,

June 23 - June 30, 2013

Saint Petersburg Area, Russia

${ }^{*}$ Speaker. 


\section{Introduction}

The soft process of multiparticle production at high energy are out of the applicability of usual QCD perturbation methods, and such processes are studied within semiphenomenological models. The parton-string approach is one of the models, that provide reasonable explanation of major experimental effects, such as energy dependence of multiplicity and flattening of its rapidity distribution [1, 2], multiplicity fluctuations and long-range correlations [3, 4]. It the last case effects of the interactions between several strings is important $[3,5]$. The approach is equally applicable as for pp, as $\mathrm{pA}$ and AA collisions.

We developed parton-string Monte Carlo model for pp, pA and AA collisions [6, 7], which include formation of strings and their and interactions in form of string fusion, as well as energymomentum conservation in the description of elementary nucleons interaction. Latter is peculiarly important in high energy interactions with nuclei, as it reduce the multiplicity and number of binary nucleon collisions significantly $[8,9,7]$.

The purpose of the present work is to perform systematic parameter turning of the MC model from experimental data on multiplicity yields in $\mathrm{pp}, \mathrm{p}-\mathrm{Pb}$ and $\mathrm{Pb}-\mathrm{Pb}$ collisions at high energy, study the sensitivity of the parameters to these observables and to obtain some constrains on the relevant physical quantities.

\section{Monte Carlo model description}

The present model $[6,7]$ is based on the partonic picture of nucleon collisions. Each nucleon supposed to consist of a valence quark-diquark pair and certain number of sea quark-antiquark pairs (see Fig. 1). The number of pairs is distributed according to Poisson low. The total momentum of a nucleon is shared between partons according to exclusive distribution [6]:

$$
\rho\left(x_{1}, \ldots x_{N}\right)=c \cdot \prod_{j=1}^{N-1} x_{j}^{-\frac{1}{2}} \cdot x_{N}^{\alpha_{N}} \cdot \delta\left(\sum_{i=1}^{N} x_{i}-1\right)
$$

which is in correspondence with inclusive distributions of the momentum fraction used in $[1,2]$. Here the valence quark is labeled by $N-1$, diquark $-N$, and the rest numbers correspond to quarkdiquark pairs; $\alpha_{N}=3 / 2$ with probability of $2 / 3$ (ud-diquark configuration), and $\alpha_{N}=5 / 2$ with probability of $1 / 3$ (uu-diquark configuration).

An elementary interaction is realized in the model of colour dipoles [10,11]. The transverse coordinates of the dipoles are generated according to Gaussian distribution, with mean-squared transverse radius $r_{0}$. It is assumed that each quark-diquark and quark-antiquark pair forms a dipole; and the probability amplitude of the collision of two dipoles from target and projectile is given by:

$$
f=\frac{\alpha_{s}^{2}}{2} \ln ^{2} \frac{\left|\vec{r}_{1}-\vec{r}_{1}^{\prime}\right|\left|\vec{r}_{2}-\vec{r}_{2}^{\prime}\right|}{\left|\vec{r}_{1}-\vec{r}_{2}^{\prime}\right|\left|\vec{r}_{2}-\vec{r}_{1}^{\prime}\right|}
$$

where $\left(\vec{r}_{1}, \vec{r}_{2}\right),\left(\vec{r}_{1}^{\prime}, \vec{r}_{2}^{\prime}\right)$ are transverse coordinates of the projectile and target dipoles (see Fig. 2), and $\alpha_{s}$ - come effective constant, which value is tunable parameter of the model. After taking into account the confinement effects $[10,11]$, the probability amplitude is:

$$
f=\frac{\alpha_{S}^{2}}{2}\left[K_{0}\left(\frac{\left|\vec{r}_{1}-\vec{r}_{1}^{\prime}\right|}{r_{\max }}\right)+K_{0}\left(\frac{\left|\vec{r}_{2}-\vec{r}_{2}^{\prime}\right|}{r_{\max }}\right)-K_{0}\left(\frac{\left|\vec{r}_{1}-\vec{r}_{2}^{\prime}\right|}{r_{\max }}\right)-K_{0}\left(\frac{\left|\vec{r}_{2}-\vec{r}_{1}^{\prime}\right|}{r_{\max }}\right)\right]^{2} .
$$




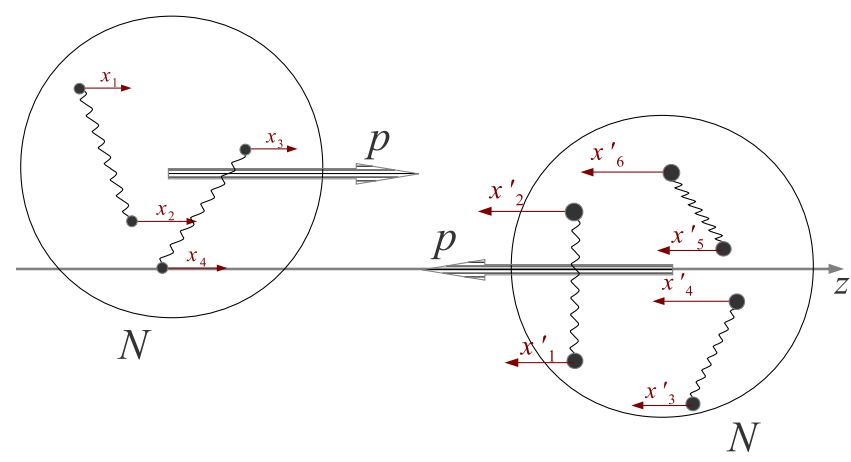

Figure 1: The schematic view of partons inside nucleons.

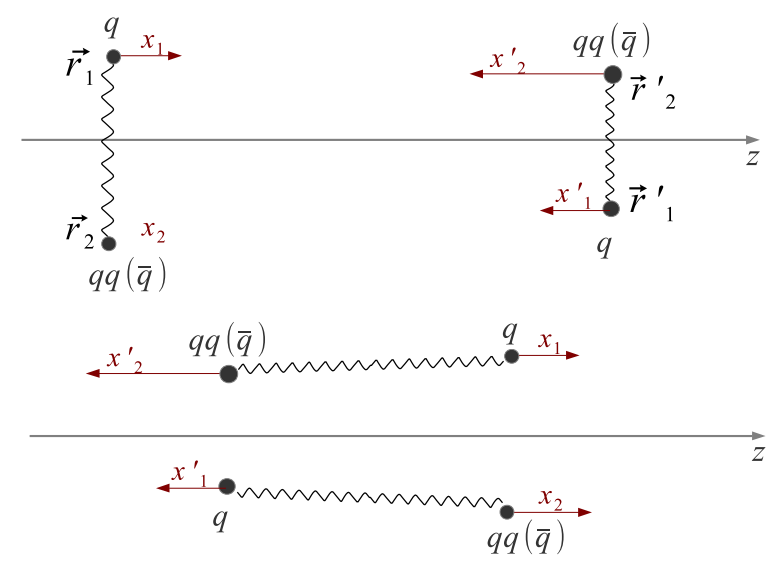

Figure 2: Monte Carlo model: color dipoles interaction and formation of the color strings.

Here $r_{\max }$ is characteristic confinement scale.

Note that according to this formulas (2.2) - (2.3), two dipoles interact more probably, if the ends are close to each other, and (others equal) if they are wide. If in Monte Carlo simulation there is a collision between two dipoles, two quark-gluon strings are stretched between the ends of the dipoles, and the process of string fragmentation gives observable particles.

An important feature of the model is that each dipole can participate in inelastic collision with color string formation only once, in order to keep the energy conservation in an elementary nucleons collision $[12,13]$.

Multiplicity and transverse momentum are calculated in the approach of colour strings, stretched between projectile and target partons, taking into account their finite rapidity width. In the calculation of the multiplicity the interaction between several string in transverse plane is taken into account, which is performed in the model of string fusion $[3,14,15]$, according to which multi- 
plicity and transverse momentum from a cluster of overlapped strings:

$$
\langle\mu\rangle_{k}=\mu_{0} \sqrt{k} \frac{S_{k}}{\sigma_{0}} \quad\left\langle p_{t}^{2}\right\rangle=p_{0} \sqrt{k}
$$

where $S_{k}$ - area, where $k$ strings are overlapping, $\sigma_{0}$ - single string transverse area, $\mu_{0}$ and $p_{0}$ - mean multiplicity and transverse momentum from one string.

Note that the amount of string fusion effect depends on the transverse radius of string: thicker the string, more the strings are overlapping. The limit of non-interacting strings corresponds to zero string radius.

\section{Parameters and fixing procedure}

The main parameters of the model are following: (i) mean number of dipoles $\lambda$; (ii) transverse mean-squared radius of nucleon $r_{0}$; (iii) confinement scale $r_{\max }$; (iv) transverse radius of string $r_{\text {str }}$; (v) mean multiplicity from single string $\mu_{0}$. We assume that only $\lambda$ depends on collision energy and require the rest of parameters to be the same at each $\sqrt{s}$.

Strategy for parameters fixing consists of two parts [6]. Firstly, using experimental data on total inelastic pp cross-section, we establish the correspondence between mean number of dipoles and collision energy $\lambda=\lambda\left(\sqrt{s} ; r_{0}, r_{\max }\right)$. This step does not include the calculation of multiplicity and, hence, does not involve $r_{\text {str }}$ and $\mu_{0}$ values. The second part of parameter fixing is intended to provide constraints on the remaining model parameters using data on multiplicity in different collision systems and wide range of energy.

We start from initial range of parameters (see Fig. 3, left):

$$
\begin{aligned}
& r_{0}: 0.4-0.7 \mathrm{fm} ; \\
& r_{\max } / r_{0}: 0.3-0.6 ; \\
& \alpha_{s}: 0.2-2.8 \\
& r_{\text {str }}: 0 \text { (no fusion), } 0.2-0.6 \mathrm{fm} ; \\
& \text { Energy range: } 53-7000 \mathrm{GeV}
\end{aligned}
$$

Parameter $\mu_{0}$ (multiplicity from one string) is fixed at intermediate LHC energy (2.36 TeV). After each step we leave only the combinations of parameters, that give results on multiplicity, consistent with experiment within error bars; otherwise the parameters combinations are discarded.

\subsection{Results for $\mathrm{pp}$ and $\mathrm{p}-\mathrm{Pb}$ collisions}

At the Fig. 3 (center) the histograms of remaining values for each parameter are shown. The results on multiplicity in pp collisions demonstrate that transverse proton radius around 0.5-0.6 fm is preferred. However, one can not make any conclusion concerning $\alpha_{s}$ and $r_{\max }$ parameters. The multiplicity in pp collisions is found also non-sensitive to the string fusion. Important constrain on the multiplicity from one string $\mu_{0}$ is obtained: $\mu_{0}$ is peaked around $1.1-1.2$. This results confirms various estimates $[5,16]$.

At the next stage the results on $d N /\left.d \eta\right|_{\eta=0}$ in minimum bias $\mathrm{p}-\mathrm{Pb}$ collisions at $5.02 \mathrm{TeV}$ were compared to experimental value [17] 16.81 \pm 0.71 . The results, shown in Fig. 3 (right), demonstrate that proton-lead collisions give more constrains on the parameters. Firstly, they exclude large 

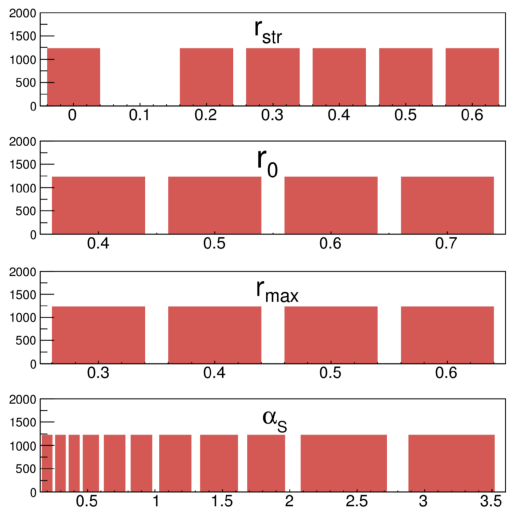

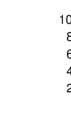
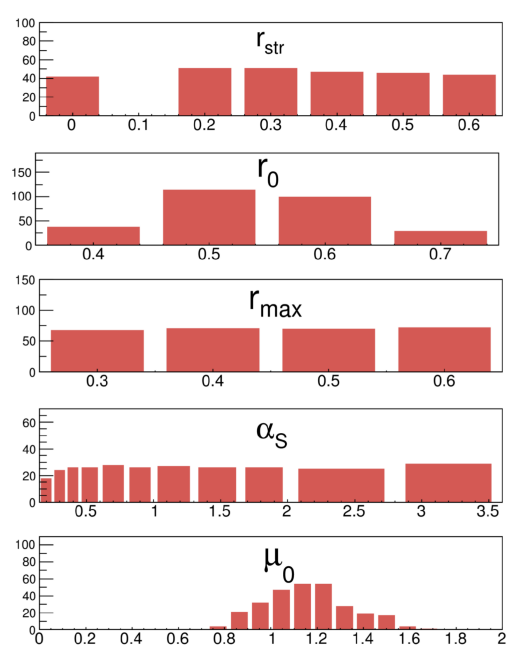
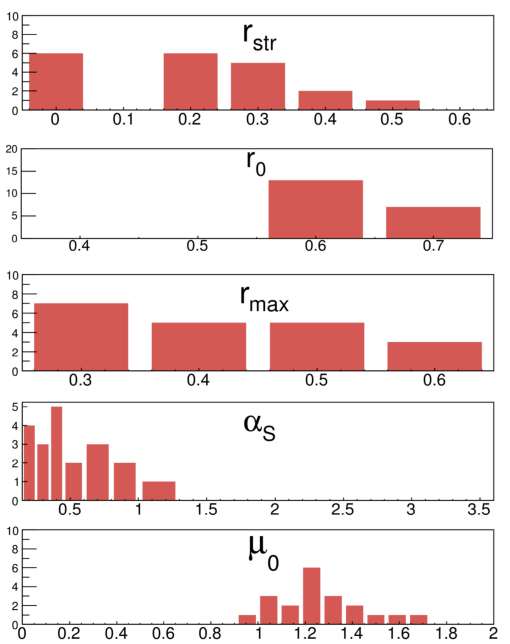

Figure 3: Monte Carlo model parameters: left - initial values, center - after accounting of pp data, right after accounting of $\mathrm{pp}$ and $\mathrm{p}-\mathrm{Pb}$ data.

string radius, leaving non-fusion case though. Secondly, small transverse radius $r_{0}$ of $0.4-0.5 \mathrm{fm}$ is excluded. Effective coupling parameter $\alpha_{s}$ is also found to be less then 1.3. The conclusion concerning $\mu_{0}$ does not change, it is still about $1.1-1.2$.

\section{Results for $\mathrm{Pb}-\mathrm{Pb}$ collisions}

The set of parameters combinations, that provide in MC model consistent description of proton-proton multiplicity in wide energy range and proton-lead multiplicity at $5.02 \mathrm{TeV}$, was used in calculation of multiplicity in $\mathrm{Pb}-\mathrm{Pb}$ collisions at $2.76 \mathrm{TeV}$. The results are shown in Fig. 4.

On can see that the shape of the multiplicity density over participant pair as a function of $N_{\text {part }}$ is reproduced in the model. All the parameters combinations give equal results in peripheral collisions, but in central they are arranged in the order of string radius. The multiplicity in nonfusion case is almost two times higher then experimental data, while the results with string radius between 0.2 and $0.3 \mathrm{fm}$ are in a good agreement to the data points.

In the Tab. 1 the parameters sets, giving best description of multiplicity in $\mathrm{PbPb}$ collisions for each $r_{\text {str }}$, are shown.

\begin{tabular}{|l|l|l|l|l|}
\hline$r_{\text {str }}$ & $r_{0}$ & $r_{\max }$ & $\alpha_{s}$ & $\mu_{0}$ \\
\hline 0 & 0.6 & 0.4 & 0.9 & 1.010 \\
\hline 0.2 & 0.6 & 0.5 & 0.4 & 1.152 \\
\hline 0.3 & 0.6 & 0.6 & 0.2 & 1.308 \\
\hline 0.4 & 0.7 & 0.3 & 0.2 & 1.626 \\
\hline
\end{tabular}

Table 1: Results of the parameter fixing in MC model

In the Fig. 5 the rapidity distribution of multiplicity in central $\mathrm{PbPb}$ collisions is shown, obtained in the model with current set of parameters, together with the ALICE experimental data 


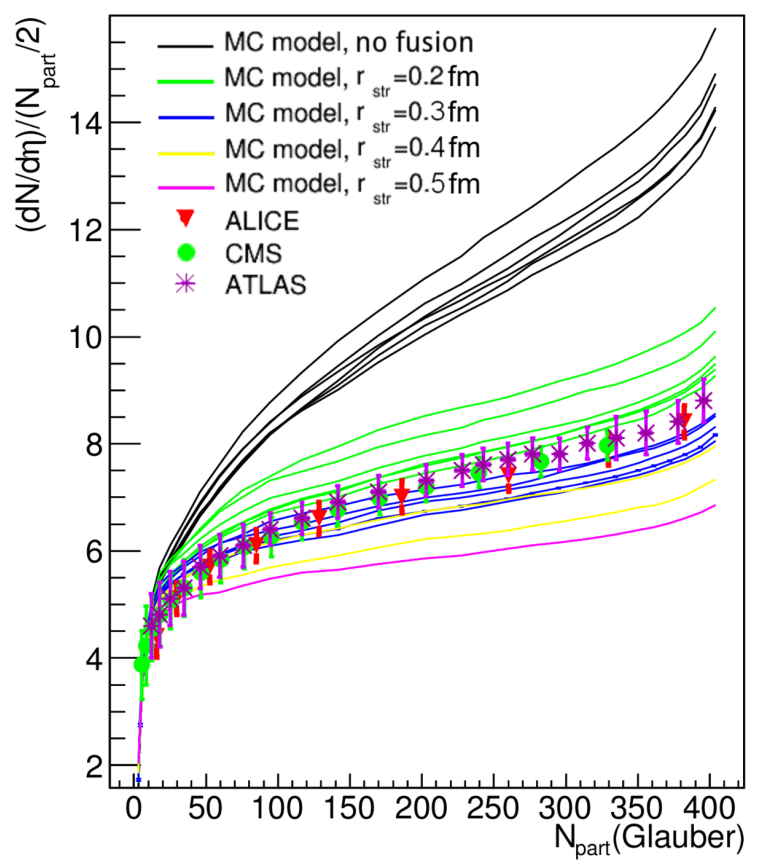

Figure 4: Charged multiplicity pseudorapidity density over half of the number of participants. Calculation in MC model (line colors correspond to different string radius) and experimental data [18, 19, 20]

[21]. The results with $r_{\text {str }}=0.2 \mathrm{fm}$ demonstrate reasonable agreement with data points within the error bars.

\section{Conclusions}

The present non-Glauber Monte-Carlo model describes multiplicity yields in wide energy range and for different colliding systems. The results on pp collisions do not fully constrain the model and one have to use different colliding systems. Multiplicity per rapidty unit from one single string is obtained at the level 1.1 - 1.2. Comparison with experiment of the dependence of multiplicity per participant on the centrality in $\mathrm{PbPb}$ collisions at $2.76 \mathrm{TeV}$ shows that good agreement is obtained only when the string fusion effects are present with the radius of string 0.2-0.3 fm.

The results of the parameter turning in the model demonstrate, that accounting of both energy conservation in the initial state and string fusion are important for the description of multiplicity yields in high energy AA collisions.

\section{References}

[1] A.B. Kaidalov, O.I.Piskunova. Zeitschrift fur Physik C 30(1):145-150, 1986

[2] G.H. Arakelyan, A.Capella, A.B.Kaidalov, and Yu.M.Shabelski. Eur.Phys.J (C), 26(1):81-90, 2002

[3] V.V. Vechernin, R.S. Kolevatov, Phys. Atom. Nucl. 70, 1797 (2007)

[4] V. Kovalenko, V. Vechernin., arXiv:1308.6618 [nucl-th] (2013). 


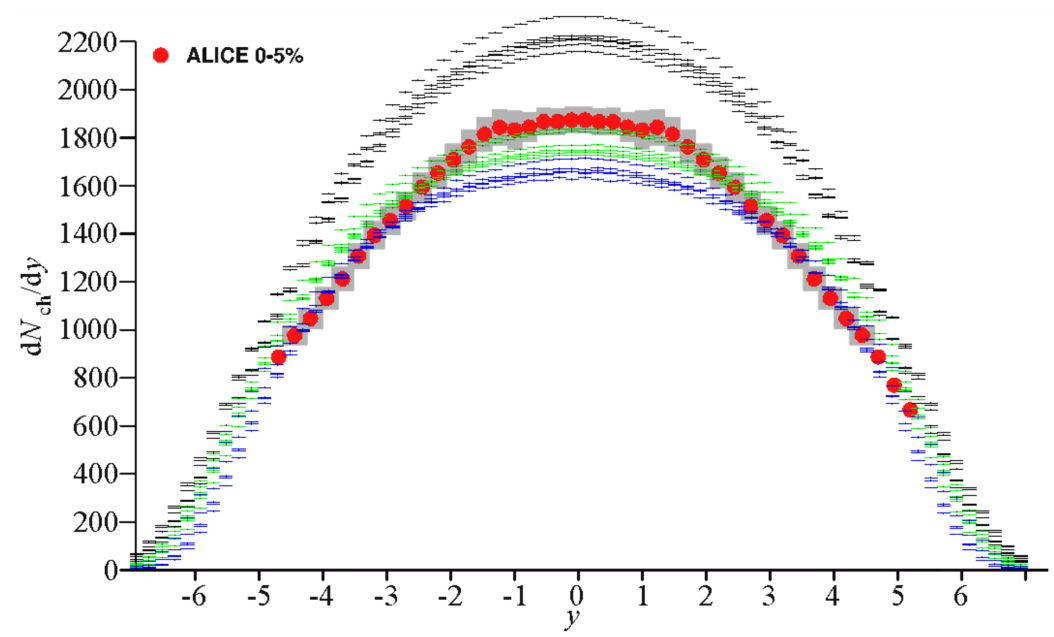

Figure 5: Rapidity distribution of multiplicity, calculated in MC model, and comparison with experimental data [21]. The meaning of the colors is the same as in Fig. 4.

[5] V.V. Vechernin, R.S. Kolevatov, Phys. Atom. Nucl. 70, 1809 (2007)

[6] V. N. Kovalenko, Phys. Atom. Nucl. 76, 1189 (2013), arXiv:1211.6209 [hep-ph]

[7] V. Kovalenko, V. Vechernin, PoS (Baldin ISHEPP XXI) 077 (2012), arXiv:1212.2590 [nucl-th]

[8] Irais Bautista, Carlos Pajares, Jose Guilherme Milhano, Jorge Dias de Deus. Phys. Rev. C 86 (2012) 034909.

[9] G. Feofilov, A. Ivanov, J. Phys. Conf. Ser. 5 (2005) 230.

[10] C. Flensburg, G. Gustafson, and L. Lonnblad, Eur. Phys. J. C 60, 233 (2009), arXiv:0807.0325

[11] G. Gustafson, Acta Phys. Polon. B 40, 1981 (2009) arXiv: 0905.2492.

[12] V. N. Kovalenko, Phys. Atom. Nucl. (submitted, 2013), arXiv:1308.1932 [hep-ph].

[13] T. Drozhzhova, G. Feofilov, V. Kovalenko, A. Seryakov, PoS (QFTHEP 2013) 052, 2013.

[14] M.A. Braun, C. Pajares, and V.V. Vechernin, Phys. Let. B 493, 54 (2000).

[15] M. Braun, R. Kolevatov, C. Pajares, and V. Vechernin, Eur. Phys. J. C 32, 535 (2004)

[16] Armesto N., Derkah D. A. and Feofilov G. A. p $p_{t}-$ multiplicity correlations in a Multi-Pomeron exchange model with string collective effects, Phys. Atom. Nucl. 71 (2008) 2087.

[17] B. Abelev et al (ALICE Collaboration), Phys. Rev. Lett. 110 (2013) 032301.

[18] K. Aamodt et al. (ALICE Collaboration), Phys. Rev. Lett. 105 (2010) 252301.

[19] G. Aad et al (ATLAS Collaboration), Phys. Lett. B 710 (2012) 363

[20] S. Chatrchyan et al (CMS Collaboration), JHEP 08 (2011) 141

[21] K. Aamodt et al. (ALICE Collaboration), Centrality dependence of the pseudorapidity density distribution for charged particles in Pb-Pb collisions at $\sqrt{s_{N N}}=2.76 \mathrm{TeV}$, Phys. Lett. B (2013), http://dx.doi.org/10.1016/j.physletb.2013.09.022, arXiv:1304.0347 [nucl-ex]. 